\title{
Colloidal suspensions confined to a film: local structure and film stability
}

\author{
D.T.Wasan ${ }^{1}$, A.Nikolov ${ }^{1}$, A.Trokhymchuk ${ }^{2,3}$, D. Henderson ${ }^{3}$ \\ 1 Department of Chemical and Environmental Engineering, \\ Illinois Institute of Technology, \\ Chicago, IL 60616, USA \\ 2 Institute for Condensed Matter Physics \\ of the National Academy of Sciences of Ukraine, \\ 1 Svientsitskii Str., 79011 Lviv, Ukraine \\ 3 Department of Chemistry and Biochemistry, Brigham Young University, \\ Provo, UT 84602, USA
}

Received January 26, 2001

\begin{abstract}
This paper summarizes recent experimental and theoretical research conducted in our laboratories on understanding the properties of colloidal suspensions confined to a film. The results of statistical mechanics modelling to explain some experiments on thinning liquid films formed from concentrated mono- and bidisperse colloidal suspensions are reported. The effect of colloidal particle size bidispersity on the local density distribution and film stability is discussed in detail.
\end{abstract}

Key words: colloidal suspensions, disjoining pressure, film stability

PACS: $82.70 . D d, 64.60 . C n, 64.75 .+g, 65.50 .+m$

\section{Introduction}

Much work has been done during the last century to investigate the properties of aqueous and organic solvents confined by dispersed nanosized colloidal particles. The last decade has shown an increased interest to the understanding of the nature of the processes carried out where the systems in which the colloidal particles themselves are under the confinement of macrosurfaces such as the walls of a container or the outer surfaces of a macroscopic bodies [liquid droplets, gas bubbles etc.] are immersed in a colloidal suspension. This is the case of numerous products such as paints, inks, food and beverages, coatings, emulsions and foams. The properties of colloidal suspensions near a single wall and the stability of the films formed from concentrated colloidal suspensions become essential for the processes such as sedimentation, lubrication, paper making, the mechanism for pollutant removal from solid surfaces, the wetting of solids by micellar solutions, etc. However, the present 
understanding of the nature of the properties of fluid films formed from molecular solvents and, particularly, from supramolecular fluids, is still far from complete.

A number of experimental techniques have been used to investigate concentrated colloidal suspensions. A new era of research towards understanding the nature of the properties of the liquid films formed from aqueous latex suspensions, surfactant micellar solutions, and microemulsions was opened when two of us discovered the layering phenomena within the film and layer-by-layer thinning of such an ordered intra-film structure [1]. Our further experiments using the differential and common interference methods, low-angle transmitted light and Kossel diffraction techniques, digital optical imaging, and video microscopy have clearly established the presence of structural forces arising from the particle layering and the in-layer particle structuring in colloidal suspensions confined between the film surfaces. These important experimental findings revealed the role of the so-called non-DLVO entropic forces such as oscillatory structural and depletion forces and thus stimulated the theoretical modelling and the studies of confined colloidal dispersions.

A big advantage of the statistical mechanics approach is that once an adequate model is formulated and an appropriate level of approximate description is established, a broad set of properties [including thermodynamics, structure and dynamics], their origin and interdependence may be examined simultaneously within the framework of the same [theoretical] "experiment". The most popular and widely used statistical mechanics model of colloidal suspensions is called the "primitive" model. This has a long history and is grounded in the fact that a small size ratio of solvent molecule to colloidal particle [typically $10^{-1}-10^{-4}$ ] could allow one to neglect the discrete nature of the solvent. Due to this, it is assumed that the solvent molecules and/or small colloidal particles ${ }^{1}$ are invisible and considered to be a continuum [the DLVO level of description of colloidal systems] on the length and the time scales of the large colloidal particles. However, the recent development of experimental and theoretical approaches and the results so obtained cast some doubts about the universality of this model. This has been discussed already [2] for the dynamics of colloidal suspensions and illustrates that the continuum has a strong effect via Stokesian friction and hydrodynamic interactions, but it is still believed that such "primitive" modelling has no influence on the static properties. At the same time the Surfaces Forces Apparatus measurements [3] indicate that even the crossed cylinders [infinitely large colloids] "recognize" the molecular scale and force acting between them has oscillatory profile with periodicity of about the diameter of solvent molecules. Statistical mechanics modellings [4,5] performed for aqueous and organic solutions with the solvent and solute taken into account explicitly confirmed these experimental observations.

We hope that even such brief insights into the state of the art of the confined colloidal suspensions sufficiently highlight the applied and basic impact of the subject and shows the existing problems and the prospects of future development. The particular goal of the present publication is two-fold. First, we wish to show an im-

\footnotetext{
${ }^{1}$ Colloidal dispersions invariably contain polydispersed particles with size ranges from several percent [polymerization processes] to hundreds of percent [emulsification processes].
} 
portant role the non-DLVO [entropic] structural forces play in properties of confined colloidal suspensions. Originally, this has been revealed and indicated by experimental investigations. We wish to show the link between statistical mechanical modelling and observations. Second, we argue that an adequate modelling of confined colloidal suspensions cannot be based on the "primitive" level. To do this, we apply the bimodal highly size-asymmetric hard-sphere model to a confined colloidal suspensions and compared with the "primitive" [one-component] model. In particular, we report the results of microscopic statistical mechanics modelling for the local density, disjoining pressure and interaction energy of the film formed from concentrated monodisperse and bidisperse colloidal suspension.

\section{Observation of the film stratification}

To observe the behaviour of thin films formed from colloidal suspensions, an original method has been developed [1]. According to this technique, the first step is to form a biconcave drop of a liquid inside a cylindrical tube with a hydrophilic inner wall. Next, the liquid is slowly sucked out of the drop through a capillary orifice in the tube wall, creating a horizontal flat film [of radius from about $5 \times 10^{-3}$ to $10^{-1} \mathrm{~cm}$ ] encircled by a biconcave liquid meniscus. The temperature is maintained constant by enclosing the glass assembly in a thermostat. Monochromatic light from the bottom of the glass cell is incident on the film surface and the light, reflected from a small portion of the thinning film area is conducted through a fiber optic probe and photomultiplier to electrometer and, finally, it is recorded in the form of photocurrent as a function of time. The film thickness, $H$, at any instant of time, can be estimated from the intensity of the photocurrent using the expression derived by Lyklema et al. [6]. The described method is a general one, appropriate for studying the films formed from micellar surfactant solutions, suspensions of latex, silica particles, and other colloidal dispersions.

Using a film formed from a micellar surfactant solution [micelle diameter, about $10 \mathrm{~nm}]$ as an example, the following phenomena have been observed. As soon as the film forms, it begins to decrease in thickness. After it becomes thinner than about ten micelle layers, the film thickness starts to change in steps [see the left panel of figure 1]. The film first rests for a few seconds in a metastable state with a uniform thickness. Then, one or more spots [with a smaller thickness than the remaining part of the film] appear and gradually increase in size. Soon, the spots cover the entire film and the film rests for several seconds in a new metastable state. Then, even darker spots appear and, after their expansion, a subsequent metastable state ensues. This process continues until the film finally reaches a stable state [of the thickness about one micelle layer] with no more step-wise changes. The calculated height of the steps is shown in the same figure and we can see that the magnitude is approximately constant for all steps [about $10.6 \mathrm{~nm}$ ], which corresponds to a micelle diameter.

The experimental data for stratifying films formed from monodispersed latex and silica hydrosol colloidal solutions, have been obtained as well. We find that the 

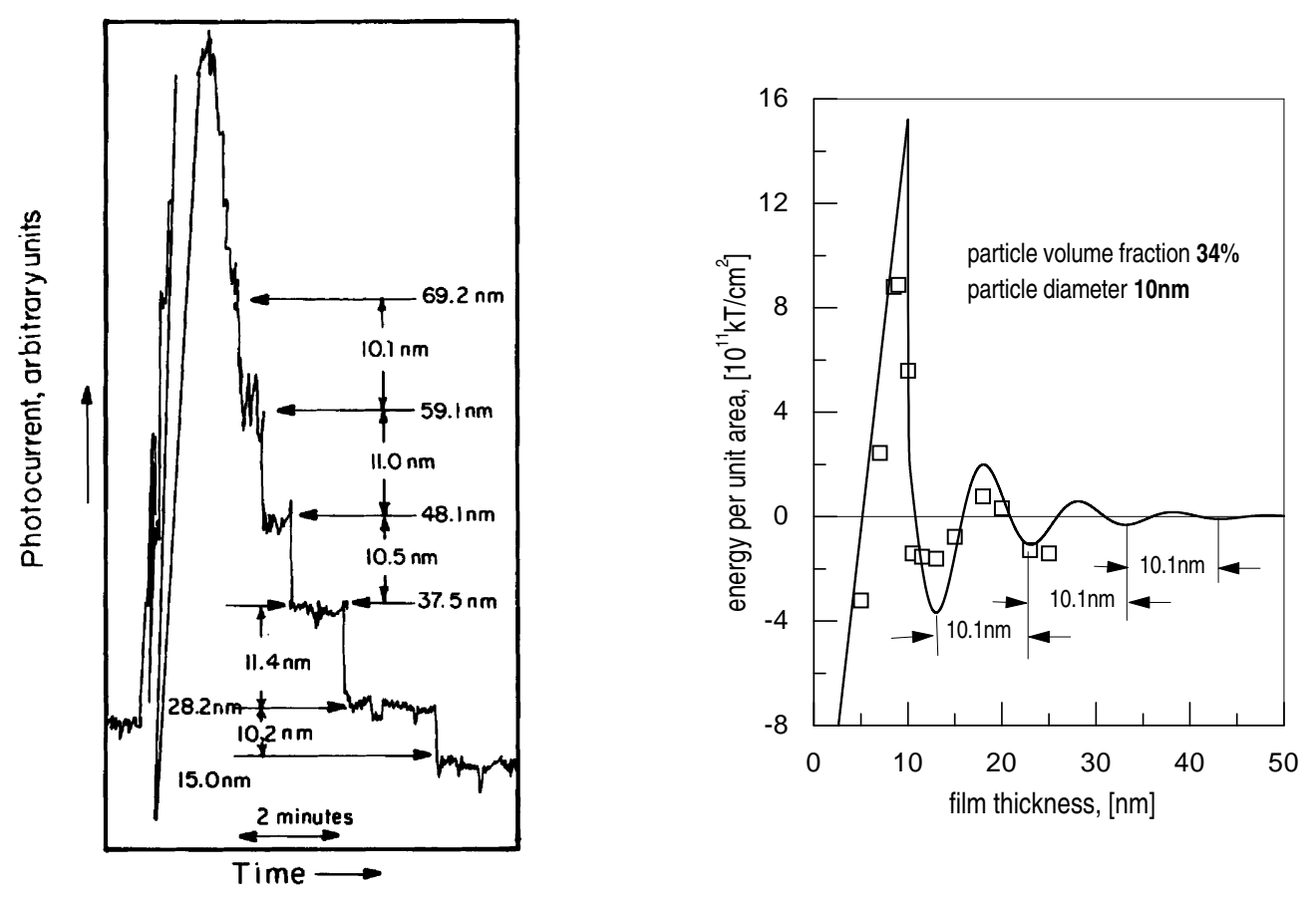

Figure 1. Left panel: Interferogram of a film formed from a micellar surfactant solution. Right panel: Film interaction energy for the film formed from a monodisperse suspension of hard-sphere particles as calculated from equation 3.8 [solid line] and predicted [7] by Monte Carlo simulations [open squares].

process of film thinning occurs with the same stepwise thickness transitions. The sizes of the transitions are uniform and are close to the effective diameters of the colloidal particles. The number of thickness transitions increases with the particle volume fraction. All this shows that film stratification is a universal phenomenon. We suggest that this stepwise film thinning results from the ordered structures of the colloidal particles that are formed within the film. The stepwise transitions are nothing more than layer-by-layer squeezing of these structures from the middle area of the film.

A direct proof of the presence of microstructures within thin films is of paramount importance. We have performed some preliminary experimental and theoretical investigations of particle structuring inside the thinning film. Experimental studies have been conducted using the low-angle transmitted light diffraction techniques with a laser wavelength of $632.8 \mathrm{~nm}$. A vertical microscopic film of the diameter of $2.5 \mathrm{~cm}$ was formed from $40 \mathrm{vol} \%$ latex suspension. On the left panel of figure 2 we present the diffraction pattern obtained. The pattern symmetry corresponds to a predominantly 2D hexagonal packing structure, i.e. colloid crystal-like in-layer structure. This hexagonal structure appeared only at film thicknesses corresponding to one and two particle layers. No such in-layer hexagonal structuring was observed for films with three particle layers. 


\section{Statistical mechanics modelling of the film stratification}

The experimental set-up described in a previous section can be imagined theoretically as a huge reservoir filled with a colloidal suspension that is a size-asymmetric two-component hard-sphere mixture of the small $(s)$ and large $(l)$ particles. The small component of this mixture has a number density, $\rho_{s}^{b}$, hard-core diameter, $d$, and its volume fraction is $\phi_{s}=\pi \rho_{s}^{b} d^{3} / 6$. The large component has a number density, $\rho_{l}^{b}$, hard-core diameter, $D$, and its volume fraction is $\phi_{l}=\pi \rho_{l}^{b} D^{3} / 6$. This is a homogeneous suspension and we refer to this as the bulk (b) suspension. To proceed, we immerse two identical freely moveable flat walls, $w$, located in the $X Y$ plane at $z=0$ and $z=H$, in a middle part of the reservoir. To describe the interaction of the suspension species with the walls, we used a hard-core particle-wall potential, $\varphi_{i w}(z)$, in a form which means that the closest approach to the wall surface occurs when the geometric centers of fluid particles are located at $z=d / 2[s$ component $]$ and at $z=D / 2[l$ component $]$. In such a reference frame, $H$ is the actual thickness of the film, i.e. thickness $H / D=1$ corresponds to one large particle layer.

The main statistical mechanics tool which we applied in the present study to obtain theoretical results is based on the integral equation (IE) theory approach, although some Monte Carlo (MC) computer simulations data [to prove our theoretical approximations] are reported as well. To adjust the IE approach to the model set-up, we start from the Ornstein-Zernike (OZ) relation between the total and direct correlation functions written for the three-component hard-sphere mixture of $s, l$ and $w$ species. The wall spheres have number density, $\rho_{w}^{b}$, and diameter, $2 R$. The two flat walls appear in the theory as a result of the so-called "wall limit" when the number density of the $w$ species tends to zero $\left[\rho_{w}^{b} \rightarrow 0\right]$ whereas their diameter tends to infinity $[2 R \rightarrow \infty]$. This leads us to the set of the three OZ equations of the form:

$$
\begin{aligned}
h_{i j}\left(r_{12}\right)-c_{i j}\left(r_{12}\right) & =\sum_{n=S, L} \rho_{n}^{b} \int h_{i n}\left(r_{13}\right) c_{n j}\left(r_{32}\right) \mathrm{d} \mathbf{r}_{3}, \\
h_{i w}\left(r_{12}\right)-c_{i w}\left(r_{12}\right) & =\sum_{n=S, L} \rho_{n}^{b} \int h_{i n}\left(r_{13}\right) c_{n j}\left(r_{32}\right) \mathrm{d} \mathbf{r}_{3}, \\
h_{w w}\left(r_{12}\right)-c_{w w}\left(r_{12}\right) & =\sum_{n=S, L} \rho_{n}^{b} \int h_{w n}\left(r_{13}\right) c_{n w}\left(r_{32}\right) \mathrm{d} \mathbf{r}_{3},
\end{aligned}
$$

where $h$ and $c$ are the pair and direct correlation functions, respectively.

Equation (3.1) is just the OZ equation for the suspension in a bulk region. The solution of equation (3.2) gives us the normalized local density distributions of the particles confined by two walls, i.e. in a film region,

$$
\rho_{i}^{\star}(H, z) \equiv \frac{\rho_{i}(H, z)}{\rho_{i}^{b}}=1+h_{i w}(H, z) .
$$

Finally, the solution of equation (3.3) can be used to obtain the effective interaction, $v(H)$, between wall solutes through the exact relation

$$
\beta v(H)=-\ln \left[h_{w w}(H)+1\right] .
$$



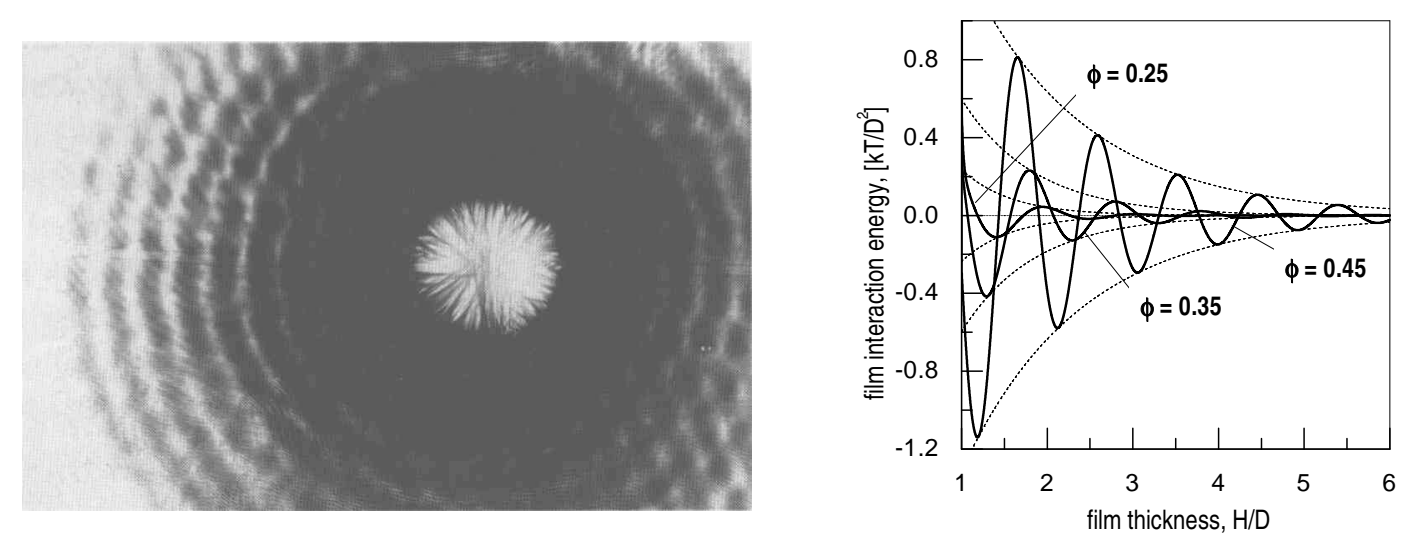

Figure 2. Left panel: Diffraction pattern from a vertical foam film formed from $40 \mathrm{vol} \%$ latex suspension. Right panel: Film interaction energy for the film formed from monodisperse suspension of hard-sphere particles at three different bulk volume fractions. The dashed curves indicate the exponential decay of the form $\pm W_{0} \mathrm{e}^{-\kappa H}$.

The question of what is an appropriate approximation for use in solving equations (3.1)-(3.3) is both controversial and unresolved. Although, the evidence [8] indicates that the Percus-Yevick (PY) closure is reasonably reliable for the problem of a thermal colloidal suspensions and molecularly smooth substrates, care must be taken in each particular case. Namely, it has been argued [9] that if $2 R \gg D$, the PY theory for total correlation function between a pair of wall particles when applied in equation (3.5), i.e. assuming $h_{w w}=h_{w w}^{\mathrm{PY}}$, leads to the result which does not obey the experimentally observed law: $v \sim R$ [3]. A qualitatively correct approximation for the total correlations between two walls has the form [4]

$$
h_{w w}(H)+1=\exp \left[h_{w w}^{\mathrm{PY}}(H)\right] .
$$

For the effective interaction between two walls this leads [5]

$$
\beta v(H)=-h_{w w}^{\mathrm{PY}}(H) .
$$

The energy per unit area between two flat walls can be obtained by differentiation of the interaction potential with respect to separation, i.e. $W(H)=\partial v(H) / \partial H$. Using the analytic PY result for the Laplace transform $\mathcal{L}\left[h_{w w}^{\mathrm{PY}}(r)\right]$, we derived a simple equation to calculate the energy of the slit-like film formed from monodisperse suspension in the form [10]

$$
\begin{aligned}
W(H) & =-P[D-H]-2 \sigma, \quad 0<H<D \\
& =W_{0} \cos (\omega H+\varphi) \mathrm{e}^{-\kappa H}+W_{1} \mathrm{e}^{-\delta(H-D)}, \quad H \geqslant D,
\end{aligned}
$$

where $P$ is the Carnahan-Starling result [11] for a suspension bulk pressure, $\sigma$ is the scaled particle theory result [12] for the surface tension near a single wall, and $W_{0}, W_{1}, \varphi, \kappa$ and $\delta$ are density dependent parameters [10]. This equation has been 
developed in a way to satisfy the known and exact statistical mechanics results and has been tested against computer simulation data.

The right panel of figure 1 displays the profile of energy per unit area between film surfaces for the film formed from monodisperse suspension as predicted by equation (3.8). The conditions, i.e. volume fraction and particle diameter are close to that of an experiment on film stratification in micellar surfactant solution. We can see that the periodicity of the energy oscillations is about one particle diameter, i.e. the same as the size of the steps in the experiments on film thinning [see left panel of the same figure 1]. Below we will find the same periodicity for the density oscillations in the film formed from monodisperse suspensions. This is a strong evidence that this oscillatory behaviour of energy is caused by the formation of well-defined particle layers inside the film and that the film will become thinner in a step-wise manner with each step being about the particle diameter.

To proceed, on the right panel of figure 2 we summarize the theoretical predictions for the effect of the particle volume fraction on both the decay length and the periodicity of oscillations of the film energy $W(H)$, as scaled with the particle diameter. It is noted, that the decay length, $1 / \kappa D$, increases upon increasing the volume fraction, while the periodicity of oscillations, $2 \pi / \omega D$, has the opposite trend. When the particle concentration in a bulk region corresponds to around a $30 \%$ volume fraction, both the decay length and periodicity of oscillation are close to the particle hard-core diameter. This theoretical result is consistent with experimental findings. In general, the dependence of both the decay length and periodicity of oscillation suggest that upon increasing colloidal particle volume fraction, the $2 \mathrm{D}$ layered structure becomes a 3D array structure.

\section{Local density distribution within the stratifying film}

Local density variations are an essential feature of the suspensions under confinement. A suspension far from a confining substrate, i.e. in the bulk region, is homogeneous and the particle local density is constant throughout. For film confinement, the local density variations depend additionally on the separation between the confining surfaces, i.e. the film thickness. In the present discussion we are focused on the case of a thick film and will pay main attention to the changes in local density for close vicinity of the film surfaces.

Two sets of theoretical "experiments" have been carried out to study the local density distributions near the film surfaces. In each we started from a bulk monodisperse suspension of only the large or only the small particles with volume fractions, $\phi_{l}=0.20$ and $\phi_{s}=0.15$, respectively. The size ratio of particle diameters, $d: D$, was chosen to be $1: 10$ and was maintained in all our calculations. A diameter of large particles, $D$, is used for scaling throughout. Since both surfaces of the film are identical, we will display only half of the film region. Such results for the normalized local densities ${ }^{2}, \rho_{i}^{\star}(z)$, are shown in figures 3 and 4 .

\footnotetext{
${ }^{2}$ Since we are discussing the thick film, we omit the film thickness, $H$, from the notation of normalized local density given by equation (3.4).
} 

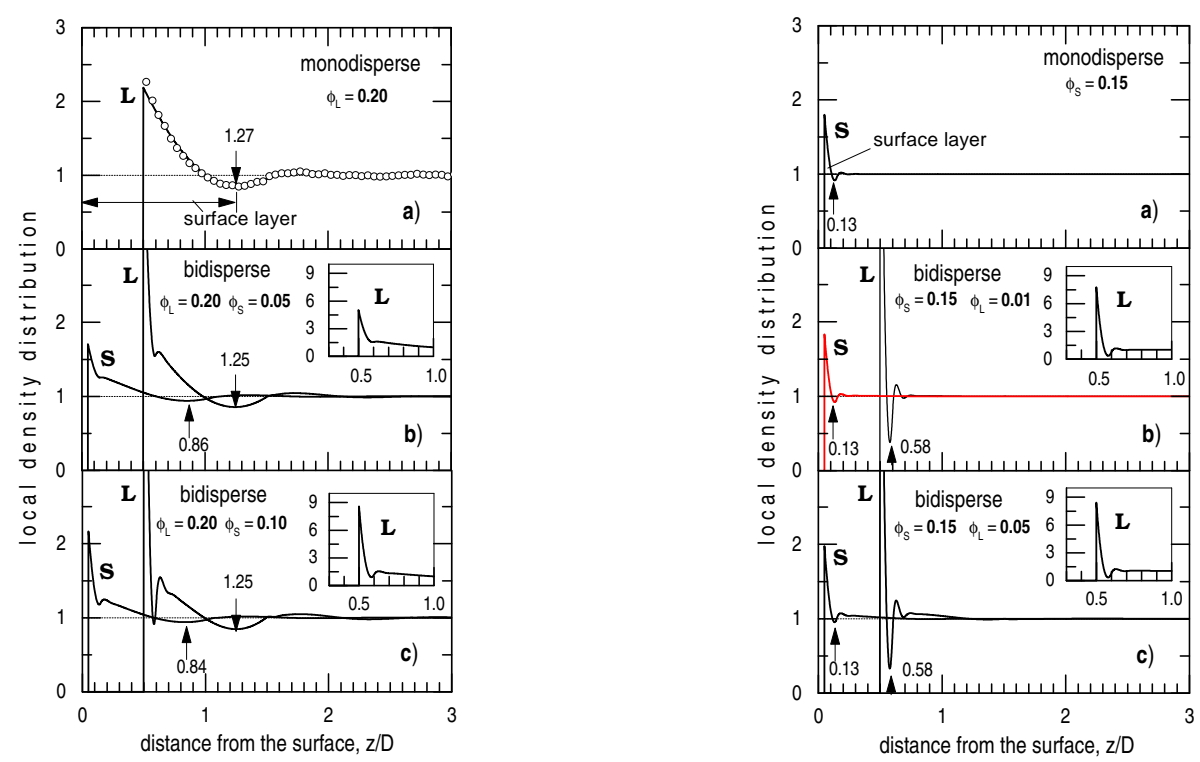

Figure 3. Left panel: Normalized local density profiles of a bidisperse suspension near a film surface. The volume fraction of the large particles is fixed at $\phi_{l}=$ 0.20 and volume fraction of the small particles changes. The open circles in part (a) correspond to MC data. The arrows indicate the positions of corresponding density minima. Right panel: The same as in left panel, but volume fraction of the small particles is fixed at $\phi_{s}=0.15$ and volume fraction of the large particles changes.

The initial fragments [parts (a)] of the left and right panels of figure 3 present the local density distributions of pure suspensions of the large and of the small particles at bulk volume fractions $\phi_{l}=0.20$ and $\phi_{s}=0.15$, respectively. Such monodisperse adsorbates are found to form a layer structure near the film surfaces: the first [or surface] layer and the second layer are separated by well-defined minima at $z=1.27 D$ [for a film from the suspension of large particles] and $z=0.13 D$ [in the case of small particles]. The maxima of the surface layers are centered at $z=0.5 D$ and $z=0.05 D$, respectively, i.e. on the film surfaces. The surface layers are diffuse, i.e. not well-ordered and, as a consequence, their thickness is about $1 \frac{1}{2}$ diameter of the constituent particles. These density distributions reflect the well-known [3] particle layering phenomena in a monodisperse film. The PY approximation reproduces this structure rather well as can be seen from the comparison with computer simulations data presented on part (a) of the left panel as well.

The transformations of the local density of the large particles, caused by changing the composition of the bulk suspension from $\phi_{l}=0.20 ; \phi_{s}=0$ to $\phi_{l}=0.20 ; \phi_{s}=$ 0.10 , are displayed on the remaining fragments of the left panel of figure 3 . When the bulk volume fraction of the small particles rises to $\phi_{s}=0.05$ [part (b)], the film surface contact density of the large particles is jumped over the bulk value by a factor of up to approximately 5 and becomes two times higher than in the case of a monodisperse suspension [part (a)]. Further increasing of the volume fraction of the small species in a bulk region to $\phi_{s}=0.10$ [part (c)] causes more large particles 

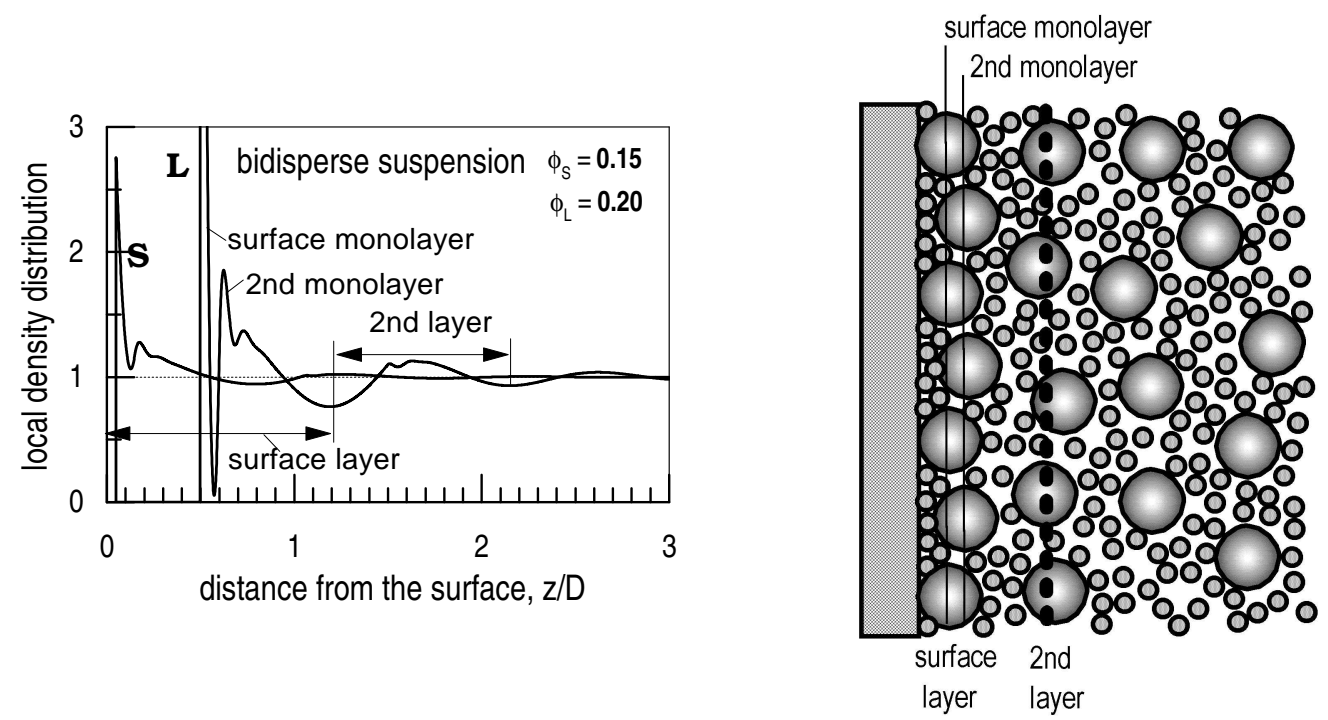

Figure 4. Left panel: The same as in figure 4, but the volume fractions of both the large and the small particles are fixed at $\phi_{l}=0.20$ and $\phi_{s}=0.15$, respectively. Right panel: Pictorial two-dimensional interpretation of bidisperse suspension ordering near a film surface.

to be adsorbed on the film surface giving rise to the formation of a thin surface sublayer, which we recognize below [see figure 4] as a surface monolayer of the large particles. A close inspection of $\rho_{l}^{\star}(z)$ near the film surface reveals that, indeed, the amount of large species located directly on the surface is increased, however, their number density in the entire surface layer ${ }^{3}$ has not been affected and remains that for the monodisperse case [part (a)]. At the same time, when the volume fraction of the small species increases to $\phi_{s}=0.15$ [figure 4], the first maximum of $\rho_{l}^{\star}(z)$ totaly splits into a well-defined monolayer located directly on the film surface and one or two [not so pronounced but clearly seen] thin sublayers. Meanwhile, with an increase of the concentration of the small particles in a bulk region, also the second layer of the large particles near a film surface becomes more pronounced. As for the small particles, they fill the remaining free space on and in the vicinity of the film surface extended up to 8-9 small particle diameters ${ }^{4}$. The nearly monotonic shape and slow decay of $\rho_{s}^{\star}(z)$ in this region indicate a high packing of the small particles [the local density is higher than in the bulk region] but without evidence for particle structuring with respect to the wall, except their surface layer.

Finally, the remaining fragments of the right side of figure 3 present the evolution of the local density of the small species near a film surface due to changing the suspension composition in a bulk region from $\phi_{s}=0.15 ; \phi_{l}=0$ to $\phi_{s}=0.15 ; \phi_{l}=$ 0.05 . The addition of only $1 \%$ of the large particles $\left[\phi_{l}=0.01\right]$ to the bulk region

\footnotetext{
${ }^{3}$ The integral over separation from the wall, $z=0$, to the first minimum of the density profile at $z=1.25 D$.

${ }^{4}$ This follows from the position of the first minima of the small particle density profiles, which is located at $z \approx 0.8 D$.
} 
does not affect the local density of the small particles near the film surface [part (b)] but clearly indicates the formation of a surface localized monolayer of the large particles being almost identical to that we observe in figure 4 at a high concentration of the large particles $\left[\phi_{l}=0.20\right]$ in the bulk region. We turn attention to this fact, since no layering phenomena are observed when a monodisperse suspension of only the large particles at a low bulk volume fraction, such as $\phi_{l}=0.01$, is used. This is a strong argument of the prominent role the small particle background play in bidisperse suspensions [or solvent molecules in a monodisperse suspensions] to create the conditions of a strong and pure entropic attraction within the large particles and between the large particles and film surface. A well-defined second sublayer of the large particle is also seen in the same part (b), i.e. the large particles are adsorbed on a surface layer of the small species. Further increase of the concentration of the large particles in a bulk region to $\phi_{l}=0.05$ [part (c)] affects only this second sublayer, which becomes less organized, indicating the destruction [by the surface-attracted large particles] of the surface layering of the small species, that has been formed in their pure suspension [part (a)]. In such an environment, the small particles prefer to be adsorbed on the surface of the large spheres and fill the cavities made by the large spheres and the film surface, forming an effective surface coverage. A further increase of the volume fraction of the large particles in a bulk region brings the film to the state shown in figure 4 with $\phi_{l}=0.20$.

\section{Film disjoining pressure and interaction energy}

The properties of a major interest for the study of film stability are the film disjoining pressure and the energy of interaction between film surfaces. According to the definition [3], the disjoining pressure, $\Pi(H)$, is a normal pressure inside the film, measured relative to the pressure outside the film, i.e. to the pressure in a bulk region:

$$
\Pi(H)=P^{\mathrm{N}}(H)-P^{\mathrm{B}} .
$$

The normal pressure, $P^{\mathrm{N}}(H)$, is the pressure exerted on the inner side of film surfaces [in the direction perpendicular to the surfaces] by the film particles. This can be evaluated from the local density profiles, $\rho_{i}(H, z)$, and as a function of film thickness is given by

$$
\beta P^{\mathrm{N}}(H)=-\beta \int_{0}^{H}\left(\frac{\partial U_{s w}(z, H)}{\partial z} \rho_{s}(H, z)+\frac{\partial U_{l w}(z, H)}{\partial z} \rho_{l}(H, z)\right) \mathrm{d} z .
$$

The bulk pressure, $P^{\mathrm{B}}$, is one of the parameters that determine the thermodynamic state of the suspension in a bulk region. This pressure is the limiting value of the normal pressure in the case of infinitely thick film, i.e. $P_{\mathrm{B}} \equiv P^{\mathrm{N}}(H \rightarrow \infty)$. In particular, to calculate the bulk pressure we used a surface separation of ten large particle layers. This provides us with a well-defined homogeneous region at the middle of the film. On the other hand, according to Derjaguin formalism [3], the disjoining pressure can also be measured by displacing one of the film surfaces by 
a distance $\Delta H$. Then, the energy [work] per unit area to bring the surfaces from infinity to the separation $H$, can be calculated as

$$
W(H)=\int_{H}^{\infty} \mathrm{d} H^{\prime} \Pi\left(H^{\prime}\right) .
$$

The equation (5.3) is an important expression because it links the calculated properties, i.e. local density distributions and normal pressure with the experimental measurements of the energy of the interaction between film surfaces. We also wish to remind that colloidal suspensions in the present study are modeled by hardsphere-like particles, i.e. suspensions are a thermal, and both the disjoining pressure and the energy of interaction between film surfaces are of pure entropic origin. They result only from a distinct structural ordering of colloidal particles in a bulk and film regions discussed above.

Figure 5 displays the profiles of the resulting structural disjoining pressure and structural interaction energy between film surfaces as functions of a film thickness for monodisperse and bidisperse suspensions. A sharp peak in the disjoining pressure exerted by a monodisperse film near a thickness of one particle diameter is apparent: as the film thickness is reduced to about one particle diameter, it becomes very difficult to squeeze the particles in the last layer out of the film. This force is purely steric. Thus, the disjoining pressure rises to a high value that corresponds to the repulsive stabilization energy barrier. Once the film thickness is reduced to a value slightly smaller than one large particle diameter, there will be no particles inside the film, the steric force disappears, and the disjoining pressure drops to zero [or even less] leading to a depletion attraction in the energy profile and to film floculation. Qualitatively similar but quantitatively quite different, i.e. a more sharp and twice higher peak is observed for a film formed from bidisperse suspension. Surprisingly, when the film thickness is reduced to a value slightly smaller than one large particle diameter, the pressure inside the film does not drop significantly and the resulting disjoining pressure is still positive and large [in our particular case it is the same order as stabilization disjoining pressure in a monodisperse film at the thickness of one large particle diameter]. Consequently, the "depletion" energy is repulsive and the film remains stable.

This will cause different trends in the film stratification process. In particular, the film thicknesses that correspond to mechanical equilibrium $[\Pi(H)=0]$ will be different. The detailed analysis of the film energy and disjoining pressure profiles vs film thickness reveals that mechanical equilibrium between the film formed from monodisperse suspension and the monodisperse bulk reservoir with volume fraction, $\phi=0.35$, is observed at film thicknesses, $H=3.2 D ; 2.2 D ; 1.2 D$ while in the case of a bidisperse film and bidisperse bulk reservoir with the same volume fraction, i.e. $\phi=\phi_{l}+\phi_{s}=0.35$ it is reached at $H=2.85 D ; 1.95 D ; 1.13 D$ and additionally at $H=1.02 D$. These thicknesses correspond to a minima of film energy and will define the metastable film thicknesses that could be measured experimentally by light interferometry. Note, that not all zeroes of disjoining pressure will correspond to a metastable film thicknesses. In general, the film of thicknesses $H$ will be metastable if a condition: $\partial \Pi / \partial H<0$ is fulfilled. 

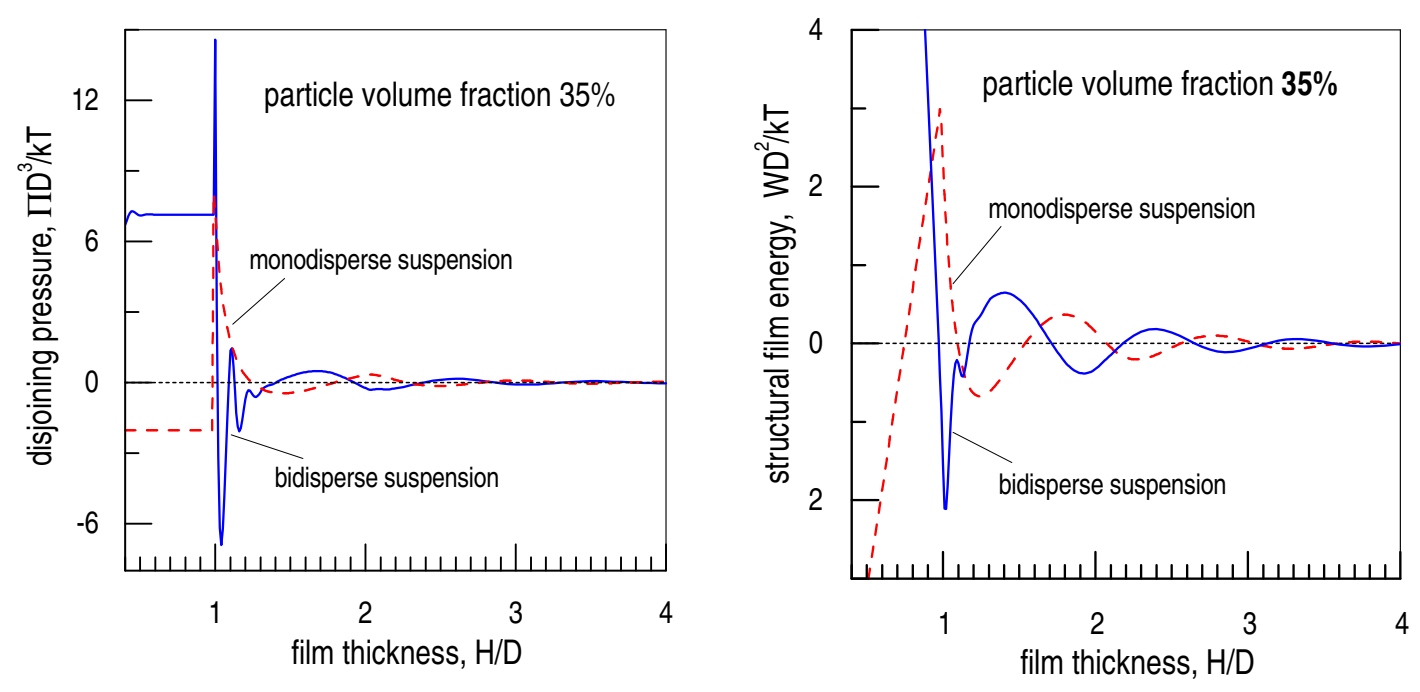

Figure 5. Structural disjoining pressure [left panel] and structural interaction energy between film surfaces [right panel] of a films formed from monodisperse [dashed line] and bidisperse [solid line] suspensions. The particle volume fractions in both cases are $35 \%$.

\section{Conclusions}

A model film formed from a bimodal hard-sphere-like suspension with particle size ratio 1 : 10 confined between two structurless hard walls has been investigated. We have confirmed the conclusions, obtained from the observation, that the presence of the small particles becomes crucial and has a strong impact on the ordering of the large species near the planar wall. The most striking prediction from the present study is concerned with the formation a surface-localized monolayer of the large particles. Perhaps we should note a very evident correlation between the bidisperse composition, i.e. $\phi_{l}$ and $\phi_{s}$, when the surface monolayer of the large particles becomes well-defined [the dip developed in $\rho_{l}^{\star}(z)$ at $z=0.58 D$ is well-defined], for the bidisperse compositions at which a surface crystalline phase was observed [13].

As the number density of the large particles in the monolayer is increased, a question is raised: What is the lateral structure of the large particles on and near the film surface? The singlet level of integral equation theory, that we employed in the present study, does not give information about the in-layer correlation functions and, obviously, we cannot definitely answer the above question. Nevertheless, we still can obtain some information from the local density variations near the film surface. In particular, the estimated number density of the large species in a monolayer is $\approx 2$ per surface area of ten large particles. Thus, it is very unlikely that crystalline order can be formed within the monolayer of the large particles. At the same time, the surface coverage provided by the small particles in the vicinity of the wall is quite high $[\approx 240$ small particles per surface area of one large particle for the composition conditions corresponding to figure 4]. In conjunction with the strong large particlefilm surface entropic attraction, such a density distribution of the small particles 
will limit the large species movement on the wall forming an effective atomically structured or corrugation-like coverage of the film [adsorbent] surface. This allows us to speculate that the remaining large particles, that are adsorbed on such a structured substrate, will grow into the crystallites in the direction normal to the film surface that are observed experimentally. In this case the formation of the surface monolayer of the large particles, revealed by the present calculations, might be the necessary condition and physical reason that can explain why surface crystallization and surface phase separation foreshadows phase separation in the bulk bidispersion.

The structural disjoining pressure and structural energy of a bidisperse film as the functions of film thickness have oscillatory decay profiles but the periodicity and phase of oscillations are more complex then for a film formed from a monodisperse suspension. When the film is thick, the periodicity of the oscillations is of the order of one large particle diameter, as it for a monodisperse film, but the phases of oscillations are almost opposite, i.e. metastable thicknesses for monodisperse and bidisperse films are different. Due to the adsorption of the small particles on the film surfaces and on the surfaces of the large particles as well, at the film thicknesses about one large particle layer, the pressure exerted by the film and the film energy start to oscillate with a periodicity of the small particle diameter. This results in a novel [with respect to the film formed from a monodisperse suspension] energy minima and zero of disjoining pressure which have been observed at the film thickness only slightly larger than one layer of the large particles, which is rather stable due to the very low decrease in film energy and very high increase in disjoining pressure.

\section{Acknowledgements}

AT and DH were supported in part by NSF (Grant No. CHE98-13729). DTW and AN were supported in a part by a DOE grant.

\section{References}

1. Nikolov A.D., Wasan D.T. Ordered micelle structuring in thin films formed from anionic surfactant solutions. I. Experimental. // J. Colloid Interface Sci., 1989, vol. 133, No. 1, p. 1-12.

2. Biben T., Hansen J.-P., Löven H. Some aspects of the Statistical Mechanics of concentrated colloidal suspensions. - In: Structure and Dynamics of Strongly Interacting Colloids and Supramolecular Aggregates in Solution. Kluwer Academic Publishers, 1992, p. 23-37.

3. Israelachvili J.N. Intermolecular and Surface Forces. New-York, Academic Press, 1992.

4. Henderson D. An explicit expression for the solvent contribution to the force between colloidal particles using a hard sphere model. // J. Colloid Int. Sci., 1988, vol. 121, No. 2, p. 486-490.

5. Trokhymchuk A., Henderson D., Wasan D.T. A molecular theory of the hydration force in an electrolyte solution. // J. Colloid Int. Sci., 1999, vol. 210, No. 2, p. 320-331.

6. Lyklema J., Scholten P.C., Mysels K.J. Flow in thin liquid films. // J. Phys. Chem., 1965, vol. 69 , No. 1, p. 116-123. 
7. Dickman R., Attard P., Simonian V. Entropic forces in binary hard sphere mixtures: Theory and simulations. // J. Chem. Phys., 1997, vol. 107, No. 1, p. 205-213.

8. Biben T., Bladon P., Frenkel D. Depletion effects in binary hard-sphere mixture. // J. Phys.: Condens. Matter, 1996, vol. 8, No. 50, p. 10799-10821.

9. Experimental measurement [3] for the force $F$ indicates that $F(r)=\partial v(r) / \partial r \sim$ $R$. Since exact relation (3.5), we obtain the conclusion that in the "wall limit" $\ln \left[h_{w w}(H)+1\right] \sim R$. In particular, for pure suspension of the large particles at the wall contact, $H=0$, the PY result is $h_{w w}^{\mathrm{PY}}(0)+1=(R / D) \frac{3}{2} \phi_{l} /\left(1-\phi_{l}\right)^{2}$ that would predict $W \sim \ln R$.

10. Trokhymchuk A., Henderson D., Nikolov A., Wasan D.T. A simple calculations of structural and depletion forces for fluids confined in a film. // Langmuir, 2001 (in press).

11. Carnahan N.F., Starling K.E. Equation of state for nonattracting rigid spheres. // J. Chem. Phys., 1969, vol. 51, No. 2, p. 635-636.

12. Reiss H., Frisch H.L., Helfand E., Lebowitz J.L. Aspects of the statistical thermodynamics of real fluids. // J. Chem. Phys., 1960, vol. 32, No. 1, p. 119-124.

13. Kaplan P.D., Rouke J.L., Yodh A.G., Pine D.J.. Entropically driven surface phase separation in binary colloidal mixtures. // Phys. Rev. Lett., 1994, vol. 72, No. 4, p. $582-585$.

\title{
Плівки з колоїдних суспензій: локальна структура та стабільність плівок
}

\author{
Д.Т.Васан ${ }^{1}$, А.Ніколов ${ }^{1}$, А.Трохимчук ${ }^{2,3}$, Д.Гендерсон ${ }^{3}$ \\ 1 Факультет хімічної інженерії та охорони навколишнього \\ середовища, \\ Іллінойський технологічний інститут, Чикаго, IL 60616, США \\ 2 Інститут фізики конденсованих систем НАН України, \\ 79011 Львів, вул. Свєнціцького, 1 \\ 3 Факультет хімії та біохімії, Університет Брайхем Янг, \\ Прово, UT 84602, США
}

Отримано 26 січня 2001 р.

Стаття узагальнює останні експериментальні та теоретичні дослідження, проведені в наших лабораторіях, щодо пояснення властивостей колоїдних суспензій в обмежених об'ємах, зокрема колоїдних плівок. У статті представлено результати статистико-механічного моделювання експериментальних спостережень процесу звуження плівок, утворених одно- та двокомпонентними колоїдними суспензіями. Детально обговорюється вплив розмірів колоїдних частинок на розподіл локальної густини у плівках та їх стабільність.

Ключові слова: колоїдні суспензії, розклинюючий тиск, стабільність плівок

PACS: $82.70 . D d, 64.60 . C n, 64.75 .+g, 65.50 .+m$ 